\title{
Ultrasound as a useful tool to integrate the clinical assessment of nail involvement in psoriatic arthritis
}

\author{
Jessica Gutierrez-Manjarrez ${ }^{1}$, Marwin Gutierrez ${ }^{1}$, Chiara Bertolazzi ${ }^{1}$, Alfonso Alfaro-Rodriguez ${ }^{2}$, \\ Carlos Pineda ${ }^{1}$
}

${ }^{1}$ Division of Musculoskeletal and Rheumatic Disorders, Instituto Nacional de Rehabilitacion, Mexico City, Mexico

${ }^{2}$ Division of Neurosciences, Instituto Nacional de Rehabilitacion, Mexico City, Mexico

\begin{abstract}
Developing the skills to adequately assess nail lesions in psoriasis is mandatory for correct interpretation of the pathological features and to provide correct management of psoriatic patients. Although clinical assessment is part of an accurate diagnosis of nail psoriasis, recent advances in the field of imaging are generating growing interest among clinicians exploring its potential role for the assessment of nail psoriasis. We would like to address the attention to ultrasound (US), which is having an impact in different clinical scenarios such as diagnosis, prognosis, and treatment monitoring of nail involvement in psoriatic disease
\end{abstract}

Key words: psoriasis, ultrasound, nail, onychopathy.

Recognition of nail lesions in psoriasis is essential for a diagnosis and helps to provide adequate management of psoriatic patients. The basic evaluation of nail changes in psoriasis is still the evaluation carried out by an experienced clinician, but in recent years interesting advances in the field of imaging have opened up new research avenues aimed at exploring their possible role in the assessment of nail psoriasis. Particular attention has been paid to ultrasound examination (US), which is demonstrating a potential impact in different clinical scenarios such as diagnosis, prognosis, and treatment monitoring of nail involvement in psoriasis [1-4] We have read with interest the comprehensive review paper on nail psoriasis by Sobolewski et al. [5]. It represents an optimal guide for a best interpretation of the wide range of nail abnormalities in psoriatic disease in daily clinical practice.

Ultrasound seems to be an optimal technique to detect minimal morphostructural changes at the nail unit level (especially in the early stages), even to identify subclinical nail involvement [6].

Thanks to the high resolution of the US transducers, the nail unit can be depicted with detailed anatomical precision including the nail plates (with the volar and dorsal plate), nail bed (which should measure no more than
2.5 to $3 \mathrm{~mm}$ ), and nail matrix (Fig. 1A) [7--9]. In the early stages of psoriatic onychopathy, the ventral plate may present loss of its sharp hyperechoic definition, with focal irregularities and hyperechoic deposits. In the late stage, fusion of ventral and dorsal plates can be seen as a single, thickened, wavy hyperechoic layer with an inhomogeneous outline and loss of the virtual anechoic space between them, with or without increased blood flow in the nail bed, as assessed by power Doppler [10]. Additionally, the nail bed is generally thickened (Fig. 1B).

Gutierrez et al. [9] described how US may help in diagnosis, especially in cases when nail involvement may not be clinical visible (i.e. when there is only volar nail plate involvement). An interesting paper published by Acquitter et al. [10] showed by US how the detection of subclinical enthesopathy at nail level is frequent with different types of psoriasis (scalp and inverse). They showed how the different abnormalities of psoriatic nails, such as loss of the normal trilaminar appearance or nail thickening, are significantly associated with a risk of developing psoriatic arthritis (PSA).

Acosta-Felquer et al. [11] showed an association between subclinical US nail involvement and enthesitis at the distal interphalangeal joint (DIP) level in psoriasis.

Address for correspondence:

Marwin Gutierrez, Division of Musculoskeletal and Rheumatic Disorders, Instituto Nacional de Rehabilitacion, Calz Mexico Xochimilco, 24680 Mexico City, Mexico, e-mail: dr.gmarwin@gmail.com

Submitted: 3.11.2017; Accepted: 29.01.2018 
The message was that nail disease was closely associated with DIP US enthesitis, which supports the nail-entheseal pathogenesis theory at DIP level [12-14].

Moreover, Castellanos-Gonzalez et al. [15] showed that the clinical presence of onychopathy is a predictor of US enthesitis at DIP level in patients with psoriasis.

From the perspective of treatment monitoring there are very few papers supporting the potential role of US. Yamaoka et al. [16] performed a study aiming to verify US changes after biological treatment. At six months of therapy there were evident changes in the US nail abnormalities, consisting of improvement of the sonographic aspect of nail plates and reduction of nail bed thickness. Gutierrez et al. [17] also demonstrated how US can be useful for a global assessment of treatment monitoring of PsA patients. His group showed, in a cross -sectional study, that US can depict changes in skin and nail after biological treatment.

The use of US for the study of nail involvement in psoriasis patients remains a challenge but is a promising area of research for the coming years. Future perspectives must be made towards standardisation of the technique (US machine settings, type of transducers, and acquisition of images) and interpretation. This point addresses the emphasis on educational aspects aimed at uniform use and application of US in the assessment of nails. Moreover, studies exploring the reliability of US findings in nail involvement, the concurrent validity, and the feasibility in terms of time spent for US examination should also be the goals of future investigations in this topic. Recent works are depicting an interesting panorama on the possible clinical impact of US in assessing nails in psoriasis in the context of global assessment of PsA disease activity. Sometimes, nail changes are not clearly visible clinically. Additionally, to determine if there is or is not power Doppler (US finding related to the activity of the inflammatory process) within the nail bed is very difficult for the clinician. In this way US may contribute by showing findings additional to the clinical assessment and increasing the sensitivity to depict useful features to improve the global activity of PsA $[17,18]$.

On the other hand, data related to the utility of US to predict the progress of the disease are emerging [5, 10, 15]. US has shown that the central inflammatory change in PsA takes place in the entheseal compartment. This structure is present in almost every joint, but enthesitis manifests clinically in PSA especially in structures subject to major shear and stretch forces. Nail disease in PsA results from the close relationship between this structure and the enthesis of the DIP extensor. The inflammatory change begins in the entheses, affecting the nail according to the degree and site (matrix vs. nail bed) of inflammatory activity, and progresses proximally to

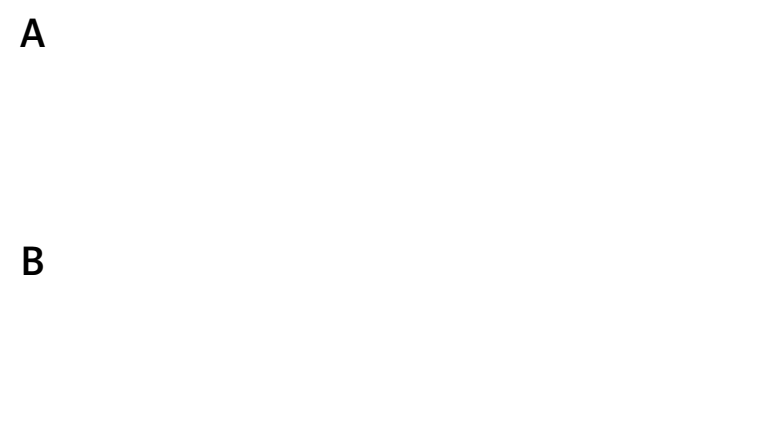

$d p$-distal phalanx; et - extensor tendon

Fig. 1. A - Nail. Healthy subject. US assessment in dorsal scan showing dorsal (arrow) and ventral plate (arrowhead), the nail bed (asterisk), and nail matrix $(\mathrm{nm})$. B - Psoriatic onychopathy. Dorsal scan. Note the fusion of ventral and dorsal plates and loss of the virtual anechoic (arrow). Moreover, the thickness of the nail bad (vertical white line), loss of the nail matrix, and the increase of power Doppler inside are evident.

affect the DIP joint. This results ultimately in the final anatomical, radiological, and clinical changes of PsA in the joints. In this view, the ability to accurately detect nail changes gives them a strategic role in the early detection of subclinical entheseal disease, progression of the anatomical damage, and in the referral and management of early PsA, thereby preventing severe, erosive, and deformative joint lesions [5].

\section{Conclusions}

Although clinical examination is still the method of reference for the assessment of nail involvement, US imaging is showing the capability to shortly become an additional tool for the initial screening of psoriatic patients at risk of nail involvement or those with psoriasis that may develop DIP arthritis.

The authors declare no conflict of interest.

\section{References}

1. Gutierrez M, Kaeley GS, Bertolazzi C, Pineda C. State of the art of ultrasound in the assessment of psoriasis and psoriatic arthritis. Expert Rev Clin Immunol 2017; 13: 439-447.

2. Delle Sedie A, Riente L. Psoriatic arthritis: what ultrasound can provide us. Clin Exp Rheumatol 2015; 33 (5 Suppl 93): $560-65$

3. Worstman X, Gutierrez M. The role of ultrasound in rheumatic skin and nail lesions: a multi-specialist approach. Clin Rheumatol 2010; 16: 14-23. 
4. Raposo I, Torres T. Nail psoriasis as a predictor of the development of psoriatic arthritis. Actas Dermosifiliogr 2015; 106 452-457.

5. Sobolewski P, Walecka I, Dopytalska K. Nail involvement in psoriatic arthritis. Reumatologia 2017; 55: 131-135.

6. Gutierrez M, Wortsman X, Filippucci E, et al. High-frequency sonography in the evaluation of psoriasis: nail and skin involvement. J Ultrasound Med 2009; 28: 1569-1574.

7. Wortsman X, Jemec GB. Ultrasound Imaging of Nails. Dermatol Clin 2006; 24: 323-328.

8. Marina ME, Solomon C, Bolboaca SD, et al. High-frequency sonography in the evaluation of nail psoriasis. Med Ultrason 2016; 18: 312-317.

9. Gutierrez M, Filippucci E, De Angelis R, et al. A sonographic spectrum of psoriatic arthritis: "the five targets". Clin Rheumatol 2010; 29: 133-142.

10. Acquitter M, Misery L, Saraux A, et al. Detection of subclinical ultrasound enthesopathy and nail disease in patients at risk of psoriatic arthritis. J Bone Spine 2017; 84: 703-707.

11. Acosta-Felquer ML, Ruta S, Rosa J, et al. Ultrasound entheseal abnormalities at the distal interphalangeal joints and clinical nail involvement in patients with psoriasis and psoriatic arthritis, supporting the nail-enthesitis theory. Semin Arthritis Rheum 2017; 47: 338-342.

12. Acosta-Felquer ML, Ferreyra Garrott L, Marin J, et al. Remission criteria and activity indices in psoriatic arthritis. Clin Rheumatol 2014; 33: 1323-1330.

13. Benjamin M, Moriggl B, Brenner E, et al. The "enthesis organ" concept: why enthesopathies may not present as focal insertional disorders. Arthritis Rheum 2004; 50: 3306-3313.

14. Tan AL, Benjamin M, Toumi H, et al. The relationship between the extensor tendon enthesis and the nail in distal interphalangeal joint disease in psoriatic arthritis - a high-resolution MRI and histological study. Rheumatology 2007; 46: 253-256.

15. Castellanos-González M, Joven BE, Sánchez J, et al. Nail involvement can predict enthesopathy in patients with psoriasis. J Dtsch Dermatol Ges 2016; 14: 1102-1107.

16. Yamaoka T, Hayashi M, Tani M, Katayama I. Value of ultrasonography findings for nail psoriasis before and after adalimumab administration. Clin Exp Dermatol 2017; 42: 201-203.

17. Gutierrez M, Di Geso L, Salaffi F, et al. Development of a preliminary US power Doppler composite score for monitoring treatment in PsA. Rheumatology 2012; 51: 1261-1268.

18. Aydin SZ, Castillo-Gallego C, Ash ZR, et al. Vascularity of nail bed by ultrasound to discriminate psoriasis, psoriatic arthritis and healthy controls. Clin Exp Rheumatol 2017; 35: 872. 